\title{
Rewarding changes in gene expression
}

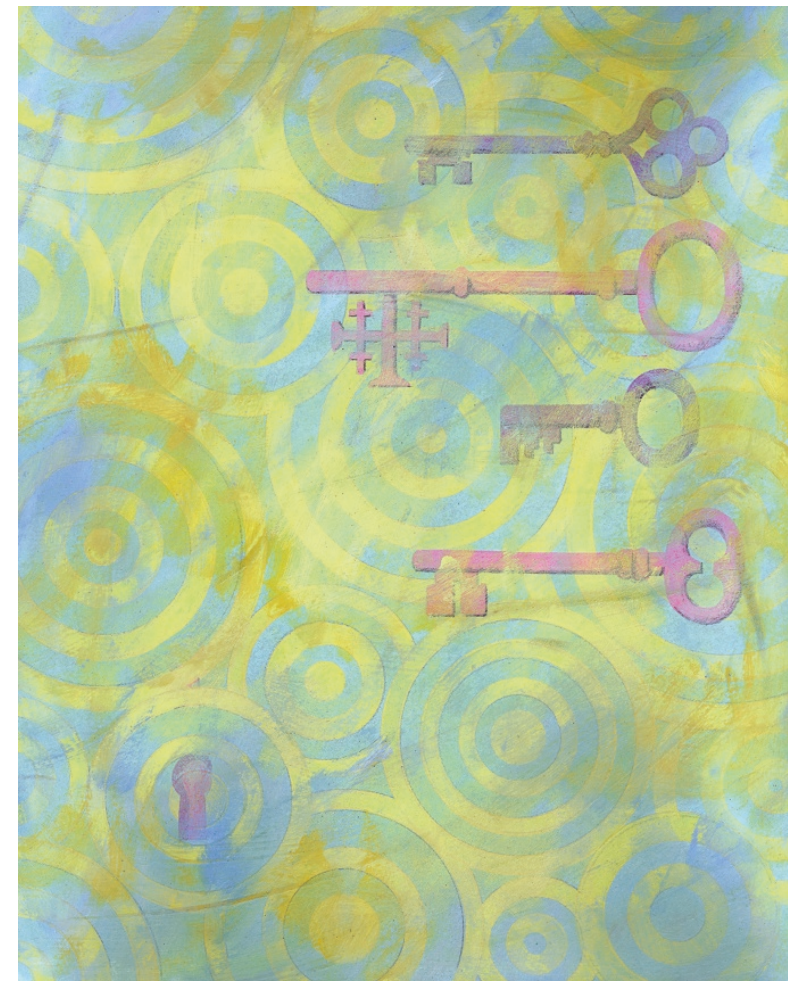

Dopamine is a key mediator of the behavioural effects of drugs of abuse and reward-driven learning, but the mechanism through which this neurotransmitter induces long-lasting effects at synapses in the striatum is poorly understood. A study in Nature now sheds light on a dopamine- triggered signalling pathway that regulates histone phosphorylation and that could, therefore, underlie the effects of rewarding stimuli on synaptic plasticity and behaviour.

Previous studies have shown that stimulation of the dopamine 1 receptor (D1R) activates dopamine- and cyclic AMP-regulated phosphoprotein with molecular weight $32 \mathrm{kDa}$

(DARPP32). In turn, DARPP32 inhibits protein phosphatase 1 (PP1), and thus affects the phosphorylation state of various synaptic proteins. However, DARPP32-mutant mice exhibit altered gene expression in response to drugs, indicating that DARPP32 also has effects in the nucleus.

DARPP32 is mostly localized in the cytoplasm, but in this study Girault and colleagues showed that administration of amphetamine, cocaine or morphine, as well as reward-driven learning, induced an accumulation of DARPP32 in the nucleus of murine striatal neurons.

In cultured striatal neurons bearing a mutation in the DARPP32 phosphorylation site Ser97, which is located close to a putative nuclear export signal, DARPP32 was found to localize in the nucleus. As Ser97 is highly phosphorylated under basal conditions, this finding suggests that dopamine inhibits nuclear export of DARPP32 by preventing the phosphorylation of Ser97. In agreement with this idea, addition of a D1R agonist to striatal slices stimulated protein phosphatase $2 \mathrm{~A}$ ( $\mathrm{PP} 2 \mathrm{~A})$ to dephosphorylate Ser97 and keep DARPP32 in the nucleus.

Importantly, accumulation of DARPP32 in the nucleus led to the phosphorylation of histone $\mathrm{H} 3$, which affects chromatin structure and therefore gene expression. Furthermore, mice bearing a mutation in the Ser97 site of DARPP32 exhibited longlasting changes in their behavioural responses to drugs of abuse, showing a decreased acute locomotor response to morphine and a reduced locomotor sensitization to cocaine. In addition, their motivation for a food reward seemed to be diminished.

Together, these findings clarify the molecular pathway by which dopamine induces changes in gene expression and behaviour towards rewarding stimuli, irrespective of whether the stimuli are natural or drug-induced.

Monica Hoyos Flight

ORIGINAL RESEARCH PAPER Stipanovich, A. et al. A phosphatase cascade by which rewarding stimuli control nucleosomal response. Nature 453, 879-884 (2008) 